IRA-International Journal of Applied Sciences

ISSN 2455-4499; Vol.04, Issue 02 (2016)

Institute of Research Advances

Pg. no. 340-350

http://research-advances.org/index.php/IRAJAS

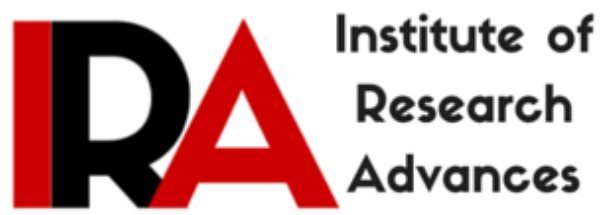

\title{
Common Fixed Points for Weak and Strong Convergence Results
}

S. C. Shrivastava

Rungta College of Engineering and Technology,

Bhilai (C.G.), India.

Type of Review: Peer Reviewed.

DOI: http://dx.doi.org/10.21013/jas.v4.n2.p15

\section{How to cite this paper:}

Shrivastava, S. (2016). Common Fixed Points for Weak and Strong Convergence

Results. IRA-International Journal of Applied Sciences (ISSN 2455-4499), 4(2), 340-350. doi:http://dx.doi.org/10.21013/jas.v4.n2.p15

(C) Institute of Research Advances

(cc) EY-NC

This work is licensed under a Creative Commons Attribution-Non Commercial 4.0 International License subject to proper citation to the publication source of the work.

Disclaimer: The scholarly papers as reviewed and published by the Institute of Research Advances (IRA) are the views and opinions of their respective authors and are not the views or opinions of the IRA. The IRA disclaims of any harm or loss caused due to the published content to any party. 
In this paper, we study the approximation of common fixed points for more general classes of mappings through weak and strong convergence results of an iterative scheme in a uniformly convex Banach space. Our results extend and improve some known recent results.

\section{INTRODUCTION}

Let $D$ be a nonempty subset of Banach space $X$. A mapping $T: D \rightarrow D$ is called asymptotically nonexpansive if for a sequence $\left\{k_{n}\right\} \subset[1, \infty)$ with $\lim _{n \rightarrow \infty} k_{n}=1,\left\|T^{n} x-T^{n} y\right\| \leq k_{n}\|x-y\|$ holds for all $x, y \in D$ and $n=1,2 \ldots T$ is also called uniform $(L-\alpha)$ - Lipschitz if for some $a>0, L>0,\left\|T^{n} x-T^{n} y\right\| \leq L\|x-y\|_{a}$ for all $x, y \in D$ and $n=1,2 \ldots$ Moreover, $\mathrm{T}$ is termed as nonexpansive if $\|T x-y\| \leq\|x-y\|$ for all $x, y \in D$ and quasi-nonexpansive if $F(T) \neq \theta$ and $\|T x-y\| \leq\|x-y\|$ for all $x \in D$ and $y \in f(T)$. The mapping $\mathrm{T}$ is called asymptotically quasinonepansive if $F(T) \neq \theta$ and there exists a sequence $\left\{k_{n}\right\}$ in $[1, \infty)$ with $\lim _{n \rightarrow \infty} k_{n}-1$, such that $\left\|T^{n} x-y\right\| \leq k_{n}\|x-y\|$ for all $x \in D, y \in F(T)$ and $n=1,2^{n \rightarrow \infty} \ldots$

Das and Debata [1] considered the following iteration scheme for two quasi-nonexpansive mappings $\mathrm{S}$ and $\mathrm{T}$ as follows:

$$
\left\{\begin{array}{l}
x_{1} \in D \\
x_{n+1}=\left(1-a_{n}\right) x_{n}+a_{n} S y_{n} \\
y_{n}=\left(1-\beta_{n}\right) x_{n}+\beta_{n} T x_{n}, \text { for all } n-1,2 \ldots
\end{array}\right.
$$

where andare in Takahashi and Tamura [4] studied the above scheme for two nanexpansive mappings.

Recently, Khan and Takahashi [2] studied the above scheme for two asymptotically nonexpansive mappings $\mathrm{S}$ and $\mathrm{T}$ through weak and strong convergence of the sequence defined by:

$$
\begin{aligned}
& {[x 1 \in D} \\
& x_{n+1}-\left(1-a_{n}\right) x_{n}+a_{n} S^{n}\left[\left(1-\beta_{n}\right) x_{n}+\beta_{n} T_{n} x_{n}\right]
\end{aligned}
$$

for all $\mathrm{n}=1,2 \ldots\left\{a_{n}\right\}$ where $\left\{\beta_{n}\right\}$ and in $[0,1]$.

Key words and phrases: Asymptotically quasi-nonexpansive mapping iterative scheme weak and strong convergence, Opial's condition, Uniform convex Banach space, Uniform (L-a)-Lipschitz, Common fixed point and continuous mappings.

2000 Mathematics Subject Classification: 47H09, 47H10, 49M05.

The first author wishes to acknowledge the financial support of Department of Science and Technology, India, made in the program year 2006-2007. Project No. SR/FTP/MS-04. 
On the other hand Bose and Laskar [5] studied the following existence theorem:

Theorem 1.1. Let $X$ be a uniformly convex Banach space and $D$ be a nonempty closed convex bounded subset of $X$ and let $S, T: D \leftarrow D$ be a continuous mappings such that for each $x, y \in D$ and $\mathrm{n}=1,2, \ldots$

$$
\begin{aligned}
\left\|S^{n} x-T^{n} y\right\| \leq & a_{n}\|x-y\|+\left(\left\|x-S^{n} x\right\|+\left\|y-T^{n} y\right\|\right) \\
& +c_{n}\left(\left\|x-T^{n} y\right\|+\left\|y-S^{n} x\right\|\right)
\end{aligned}
$$

where $a_{n}, b_{n}, c_{n} \geq 0$ and satisfying following conditions:

(i) there is an integer I such that $b_{n}+c_{n}<1 \forall n=1,2 \ldots$

(ii) $\lim _{n \rightarrow \infty} \frac{a_{n}+3 b_{n}+c_{n}}{1-b_{n}-c_{n}}=1$,

(iii) $a_{n}+2 c_{n}<1$, for at least one $\mathrm{n}$.

Then $\mathrm{S}$ and $\mathrm{T}$ have unique common fired points and it is unique as fixed points of each $\mathrm{S}$ and $\mathrm{T}$.

If we put $b_{n}=0$ then the condition (1.1) reduces to

$$
\begin{aligned}
& \left\|S^{n} x-T^{n} y\right\| \leq a_{n}\|x-y\|+c_{n}\left(\left\|x-T^{n} y\right\|+\left\|y-S^{n} x\right\|\right) \\
& \text { for all } x, y \in D \text { and } n=1,2 \ldots, \text { where } a_{n}, c_{n} \geq 0 \text { with } c_{n}<1 \text { and } \lim _{n \rightarrow \infty} \frac{a_{n}+c_{n}}{1-c_{n}}=1 .
\end{aligned}
$$

In this paper, we study the problems of approximation of common fixed points for uniform Lipschitz asymptotically quasi-nonexpansive mappings and also for the continuous mappings which satisfy the condition (1.2). Our scheme is given by the sequence $\left\{x_{n}\right\}$ in $D$ defined as follows:

$$
\left\{\begin{array}{l}
x_{1} \in D \\
x_{n+1}=\left(1-a_{n}\right) x_{n}+a_{n} T^{n} y_{n} \\
y_{n}=\left(1-\beta_{n}\right) x_{n}+\beta_{n} S^{n} x_{n}, n=1,2 \ldots
\end{array}\right.
$$

Where $\left\{\alpha_{n}\right\}$ and $\beta_{n}$ are sequences in $[0,1]$. Our results improve and extend the corresponding previously known results of khan and Takahashi [3].

\section{PERLIMINARIES}

We give the following Lemmas which we shall need in the sequel.

Lemma 2.1. [8] Let $\left\{r_{n}\right\},\left\{s_{n}\right\},\left\{t_{n}\right\}$ be three nonnegative sequence satisfying the following condition.

$$
r_{n+1} \leq\left(1+s_{n}\right) r_{n}+t_{n} \text { for all } n \in N .
$$

If $\sum_{n=1}^{\infty} s_{n}<\infty, \sum_{n=1}^{\infty} t_{n}<\infty$. Then $\lim _{n \rightarrow \infty} r_{n}$ exists.

Lemma 2.2. [9] Suppose that $X$ is a uniformly convex Banach space and $0<p \leq t_{n} \leq q<1$ for all $n \in N$. Suppose further that $\left\{x_{n}\right\}$ and $\left\{y_{n}\right\}$ are sequences of $\mathrm{X}$ such that $\limsup _{n \rightarrow \infty}\left\|x_{n}\right\| \leq r$,

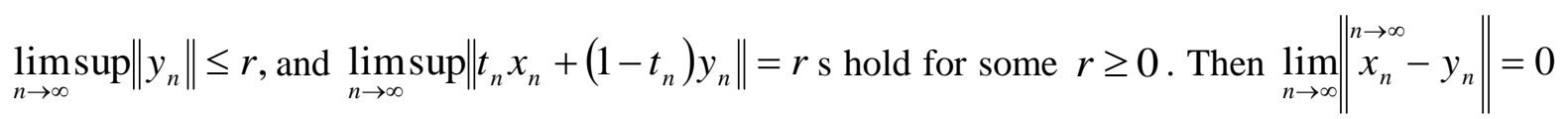


We recall that a Banach space $X$ is said to satisfy Opial's condition [12] if for any sequence $\left\{x_{n}\right\}$ in $X, x_{n} \rightarrow x$ implies that $\limsup _{n \rightarrow \infty}\left\|x_{n}-x\right\|<\limsup _{n \rightarrow \infty}\left\|x_{n}-y\right\|$ for all $y \in X$ with $y \neq x$. Moreover, we also know that a mapping $\stackrel{n \rightarrow \infty}{T}: D \rightarrow X$ is called demiclosed with respect to $y \in X$ if for each sequence $\left\{x_{n}\right\}$ in $D$ and each $x \in X, x_{n}$ and $T x_{n} \rightarrow y$ imply that $x \in D$ and $T x=y$.

Lemma 2.3. [11] Let $X$ be a uniformly convex Banach space satisfying Opial's condition and let $D$ be a nonempty of $\mathrm{D}$ into itself. Then I-t is demiclosed with respect to zero.

We shall now prove the following lemmas on the lines similar to [3]. It will be used to prove the main results.

Lemma 2.4. Let $D$ be a nonempty closed convex bounded subset of normed space $X$ and let $T, S: D \rightarrow D$ be two uniform $(L-\alpha)$-Lipschitz mappings. Define a sequence $\left\{x_{n}\right\}$ as in (1.3). Then

and

$$
\left\|x_{n+1}-T x_{n+1}\right\| \leq d_{n+1}+L\left\{2 d_{n}+L d_{n}^{\prime \alpha}+L\left(d_{n}+L d_{n}^{\prime \alpha}\right)^{\alpha}\right\}^{\alpha}
$$

$$
\left\|x_{n+1}-S x_{n+1}\right\| \leq d_{n+1}^{\prime}+L\left\{d_{n}+L d_{n}^{\prime \alpha}+d_{n+1}^{\prime}+L\left(d_{n}+L d_{n}^{\prime \alpha}\right)^{\alpha}\right\}^{\alpha}
$$

where $d_{n}=\left\|x_{n}-T_{n} x_{n}\right\|$ and $d^{\prime}{ }_{n}=\left\|x_{n}-S^{n} x_{n}\right\|$

Proof. We consider

$$
\begin{aligned}
\left\|x_{n}-x_{n+1}\right\| & =\left\|x_{n}-\left\{\left(1-\alpha_{n}\right) x_{n}+\alpha_{n} T^{n} y_{n}\right\}\right\| \\
& \leq\left\|x_{n}-T^{n} y_{n}\right\| \\
& \leq\left\|x_{n}-T^{n} x_{n}\right\|+\left\|T^{n} x_{n}-T^{n} y_{n}\right\| \\
& \leq d_{n}+L\left\|x_{n}-y_{n}\right\|^{\alpha} \\
& \leq d_{n}+L\left\|x_{n}-\left\{\left(1-\beta_{n}\right) x_{n}+\beta_{n} S^{n} x_{n}\right\}\right\|^{\alpha} \\
& \leq d_{n}+L\left\|x_{n}-S^{n} x_{n}\right\|^{\alpha}
\end{aligned}
$$

and

$$
\begin{aligned}
\|x n+1-T x n+1\| & \leq\left\|x_{n+1}-T^{n+1} x_{n+1}\right\|+\left\|T x_{n+1}-T^{n+1} x_{n+1}\right\| \\
& \leq d_{n}+1 L\left\|x_{n}+1-T^{n} x_{n+1}\right\|^{\alpha} \\
& \leq d_{n+1}+1 L\left\|\left(x_{n+1}-x_{n}\right)+x_{n}+\left(x_{n}-T^{n} x_{n}\right)+\left(T^{n} x_{n}-T^{n} x_{n+1}\right)\right\|^{\alpha} \\
& \leq d_{n+1} L\left\{\left\|x_{n+1}-x_{n}\right\|+\left\|x_{n}-T^{n} x_{n}\right\|+\left\|T^{n} x_{n}-T^{n} x_{n+1}\right\|\right\}^{\alpha} \\
& \leq d_{n+1} L\left\{d_{n}+L d_{n}^{\prime \alpha}+d_{n}+L\left\|x_{n}-x_{n+1}\right\|^{\alpha}\right\}^{\alpha} \\
& \leq d_{n+1} L\left\{2 d_{n}+L d_{n}^{\prime \alpha}+L\left(d_{n}+L d_{n}^{\prime \alpha}\right)^{\alpha}\right\}^{\alpha}
\end{aligned}
$$

Similarly, we can prove that

$$
\left\|x_{n+1}-S x_{n+1}\right\| \leq d_{n+1}^{\prime}+L\left\{d_{n}+L d_{n}^{\prime \alpha}+d_{n}^{\prime}+L\left(d_{n}+L d_{n}^{\prime \alpha}\right)^{\alpha}\right\}^{\alpha}
$$


Lemma 2.5. Let $X$ be a uniformly Banach space and let $D$ be a nonempty closed convex bounded subset of X. Let $T, S: D \rightarrow D$ be a continuous mappings satisfying condition (1.2). Given a sequence $\left\{x_{n}\right\}$ defined by (1.3). Then

$\left\|x_{n+1}-T x_{n+1}\right\| \leq \frac{1-c_{n}}{1-3 c_{n}}\left[d^{\prime}{ }_{n+1} \frac{a_{n}+c_{n}}{1-c_{n}}\left(\frac{1+a_{n}+2 c_{n}}{1-c_{n}} \cdot \frac{1+a_{n}}{1-c_{n}} d^{\prime}{ }_{n}+\frac{1+c_{n}}{1-c_{n}} d_{n}\right)\right]$

and

$\left\|x_{n+1}-S x_{n+1}\right\| \leq \frac{1-c_{n}}{1-3 c_{n}}\left[d_{n+1}+\frac{a_{n}+c_{n}}{1-c_{n}}\left(\frac{1+a_{n}+2 c_{n}}{1-c_{n}} \frac{1+a_{n}}{1-c_{n}} d_{n}^{\prime}+\frac{1-c_{n}}{1-c_{n}} d_{n}^{\prime}\right)\right]$

where $d_{n}=\left\|x_{n}-T^{n} x_{n}\right\|$ and $d_{n}^{\prime}=\left\|x_{n}-S^{n} x_{n}\right\|$.

Proof. We have

$$
\begin{aligned}
\left\|x_{n}-x_{n+1}\right\|=\| x_{n} & -\left\{\left(1-a_{n}\right) x_{n}+a_{n} T^{n} y_{n}\right\} \| \\
& \leq\left\|x_{n}-T^{n} y_{n}\right\| \\
& \leq\left\|x_{n}-S^{n} x_{n}\right\|+\left\|S^{n} x_{n}-T^{n} y_{n}\right\|
\end{aligned}
$$

From condition (1.2). we have

$$
\begin{aligned}
\left\|S^{n} x_{n}-T^{n} y_{n}\right\| \leq & a_{n}\left\|x_{n}-y_{n}\right\|+c_{n}\left(\left\|x_{n}-T^{n} y_{n}\right\|+\left\|y_{n}-S^{n} x_{n}\right\|\right) \\
\leq & a_{n}\left\|x_{n}-y_{n}\right\|+c_{n}\left(\left\|x_{n}-S^{n} x_{n}\right\|+\left\|S^{n} x_{n}-T^{n} y_{n}\right\|\right) \\
& +\left\|y_{n}-x_{n}\right\|+\left\|x_{n}-S^{n} x_{n}\right\|
\end{aligned}
$$

Then

$$
\left\|S^{n} x_{n}-T^{n} y_{n}\right\| \leq \frac{a_{n}+c_{n}}{1-c_{n}}\left\|x_{n}-y_{n}\right\|+\frac{2 c_{n}}{1-c_{n}}\left\|x_{n}-S^{n} x_{n}\right\| .
$$

From (2.2) and (2.3), we obtain

$$
\begin{aligned}
\mathrm{s}\left\|x_{n+1}-x_{n}\right\| & \leq\left\|x_{n}-S^{n} x_{n}\right\|+\frac{a_{n}+c_{n}}{1-c_{n}}\left\|x_{n}-y_{n}\right\|+\frac{2 c_{n}}{1-c_{n}}\left\|x_{n}-S^{n} x_{n}\right\| \\
(2.4) \leq & \frac{1+a_{n}+2 c_{n}}{1-c_{n}} d_{n}^{\prime} \\
\|x n+1-T x n+1\| \leq & \leq \mid x n+1-S^{n+1} x_{n+1}\|+\| T x_{n+1}-S^{n+1} x_{n+1} \| \\
\leq & d_{n+1}^{\prime}+\frac{a_{n}+c_{n}}{1-c_{n}}\left\|x_{n+1}-S^{n} x_{n+1}\right\| \\
+ & \frac{2 c_{n}}{1-c_{n}}\left\|x_{n+1}-T x_{n+1}\right\| \\
\left(\frac{1-3 c_{n}}{1-c_{n}}\right)\left\|x_{n+1}-T x_{n+1}\right\| & \leq d_{n+1}^{\prime}+\frac{a_{n}+c_{n}}{1-c_{n}}\left(\left\|x_{n+1}-x_{n}\right\|+\left\|x_{n}-T^{n} x_{n}\right\|\right) \\
& +\left\|T^{n} x_{n}-S^{n} x_{n+1}\right\| \\
& \leq d^{\prime}{ }_{n+1}+\frac{a_{n}+c_{n}}{1-c_{n}}\left(\left\|x_{n+1}-x_{n}\right\|+d_{n}+\frac{a_{n}+c_{n}}{1-c_{n}}\left\|x_{n}-x_{n+1}\right\|\right.
\end{aligned}
$$




$$
\begin{gathered}
\left.+\frac{2 c_{n}}{1-c_{n}}\left\|x_{n}-T^{n} x_{n}\right\|\right) \\
\leq d_{n+1}^{\prime}+\frac{a_{n}+c_{n}}{1-c_{n}}\left[\frac{1+a_{n}}{1-c_{n}}\left\|x_{n}-x_{n+1}\right\|+\frac{1+c_{n}}{1-c_{n}} d_{n}\right]
\end{gathered}
$$

Substituting (2.40) into (2.5), we get

$$
\left\|x_{n+1}-T x_{n+1}\right\| \leq \frac{1-c_{n}}{1-3 c_{n}}\left[d^{\prime}{ }_{n+1}+\frac{a_{n}+c_{n}}{1-c_{n}}\left(\frac{1+a_{n} 1+a_{n}+2 c_{n}}{1-c_{n}-1-c_{n}} d^{\prime}{ }_{n}+\frac{1+c_{n}}{1-c_{n}} d^{\prime}{ }_{n}\right)\right]
$$

Similarly, we can prove that

$$
\left\|x_{n+1}-S_{n+1}\right\| \leq \frac{1-c_{n}}{1-3 c_{n}}\left[d_{n+1}^{\prime}+\frac{a_{n}+c_{n}}{1-c_{n}}\left(\frac{1+a_{n}}{1-c_{n}} \frac{1+a_{n}+2 c_{n}}{1-c_{n}} d^{\prime}{ }_{n}+\frac{1+c_{n}}{1-c_{n}} d^{\prime}{ }_{n}\right)\right]
$$

Lemma 2.6. Let $X$ be a Banach space $X$ and $D$ a nonempty subset of $X$. Let $S, T: D \rightarrow D$ be two mappings such that

$$
\left\|T^{n} x_{n}-S^{n} y\right\| \leq a_{n}\|x-y\|+c_{n}\left(\left\|x-T^{n} y\right\|+\left\|y-S^{n} x\right\|\right)
$$

for all $x, y \in D$ and $n \in N$, where $a_{n}, c_{n} \geq 0$ and satisfying the following condition:

(i) $c_{n}<1$ forall $n \in N$

(ii) $\frac{a_{n}+2 c_{n}}{1-c_{n}} \leq 1$ forall $n \in N$

then

$$
\left\|T^{n} x-p\right\| \leq \frac{a_{n}+c_{n}}{1-c_{n}}\|x-p\|
$$

and

$$
\left\|S^{n} x-p\right\| \leq \frac{a_{n}+c_{n}}{1-c_{n}}\|x-p\| \text { for all } x \in D \text { and } n \in N
$$

Proof. (a) It follows from

Let $p \in F(T) \cap F(S)$.Then

$$
\begin{aligned}
\left\|T^{n} x-p\right\| & \leq a_{n}\|x-p\|+c_{n}\left(\|x-p\|+\left\|S^{n} x-p\right\|\right) \\
& \leq\left(a_{n}+c_{n}\right)\|x-p\|+c_{n}\left\|S^{n} x-p\right\|
\end{aligned}
$$

and

$$
\left\|S^{n} x-p\right\| \leq\left(a_{n}+c_{n}\right)\|x-p\|+c_{n}\left\|S^{n} x-p\right\| .
$$

Now

$$
\begin{aligned}
\left\|T^{n} x-p\right\| & \left.\leq\left(a_{n}-c_{n}\right)\|x-p\|+c_{n} \mid\left(a_{n}+c_{n}\right)\|x-p\|+c_{n}\left\|T^{n} x-p\right\|\right\rfloor \\
& \leq\left(1+c_{n}\right)\left(a_{n}+c_{n}\right)\|x-p\|+c_{n}^{2}\left\|T^{n} x_{n}-p\right\|
\end{aligned}
$$

which implies that

$$
\left\|T^{n} x-p\right\| \leq \frac{a_{n}+c_{n}}{1-c_{n}}\|x-p\| .
$$

Similarly,

$$
\left\|S^{n} x_{n}-p\right\| \leq \frac{a_{n}+c_{n}}{1-c_{n}}\|x-p\| \text { for all } x \in D \text { and } n \in N
$$




\section{MAIN RESULTS}

Theorem 3.1. Let $X$ be a uniformly convex Banach space and D be a nonempty closed convex bounded subset of Banach space $X$. Let $T, S: D \rightarrow D$ be an asymptotically quasi-nonexpansive mappings with sequence $\left\{k_{n}\right\}$ such that $\sum_{n=1}^{\infty}\left(k_{n}-1\right)<\infty$ and $F(T) \cap F(S) \neq \phi$. Define a sequence $\left\{x_{n}\right\}$ in D as (1.3) Then the following hold:

(a) $\lim _{n \rightarrow \infty}\left\|x_{n}-p\right\|=\lim _{n \rightarrow \infty}\left\|y_{n}-p\right\|$ exists.

(b) $\lim _{n \rightarrow \infty}\left\|x_{n}-T x_{n}\right\|=0=\left\|x_{n}-S x_{n}\right\|$ if $S$ and T is a uniform $(L-a)-$ Lipschitizion.

Proof. (a) Let $p \in F(T) \cap F(S)$. Then

$$
\begin{aligned}
\left\|x_{n+1}-p\right\| & =\left\|\left(1-a_{n}\right) x_{n}+a_{n} T^{n} y_{n}-p\right\| \\
& \leq\left\|x_{n}-p\right\|+\left\|T^{n} y_{n}-p\right\| \\
& \leq\left\|x_{n}-p\right\|+k_{n}\left\|y_{n}-p\right\| \\
\left\|y_{n}-p\right\| & =\left\|\left(1-\beta_{n}\right) x_{n}+\beta_{n} S^{n} x_{n}-p\right\| \\
& \leq\left(1-\beta_{n}\right)\left\|x_{n}-p\right\|+\beta_{n}\left\|S^{n} x_{n}-p\right\| \\
& \leq k_{n}\left\|x_{n}-p\right\|
\end{aligned}
$$

From (3.1) and (3.2), we get

$$
\left\|x_{n+1}-p\right\| \leq k_{n}^{2}\left\|x_{n}-p\right\|
$$

(b) Suppose $\lim _{n \leftarrow \infty}\left\|x_{n}-p\right\|=d$, for some $\mathrm{d}>0$.

Since

It follows that

$$
\left\|T^{n} y_{n}-p\right\| \leq k_{n}\left\|y_{n}-p\right\| .
$$

We observe that

$$
\underset{n \rightarrow \infty}{\limsup }\left\|T^{n} y_{n}-p\right\| \leq \limsup _{n \rightarrow \infty}\left(k_{n}\left\|y_{n}-p\right\|\right)
$$

$$
\lim _{n \rightarrow \infty}\left\|x_{n}-p\right\| \leq d \text { and } \lim _{n \rightarrow \infty}\left(T^{n} y_{n}-p\right) \leq d .
$$

Then

$$
\lim _{n \rightarrow \infty}\left\|x_{n+1}-p\right\|=\lim _{n \rightarrow \infty}\left\|a_{n}\left(T^{n} x_{n}-p\right)+\left(1-a_{n}\right)\left(x_{n}-p\right)\right\|-d
$$

From Lemma 2.2, we obtain

$$
\lim _{n \rightarrow \infty}\left\|x_{n}-T^{n} y_{n}\right\|=0
$$

Further

$$
\begin{aligned}
& \left\|x_{n}-p\right\| \leq\left\|x_{n}-T^{n} y_{n}\right\|+\left\|T^{n} y_{n}-p\right\| \\
& \leq\left\|x_{n}-T^{n} y_{n}\right\|+k_{n}\left\|y_{n}-p\right\|
\end{aligned}
$$

Gives that

$$
d \leq \liminf _{n \rightarrow \infty}\left\|y_{n}-p\right\| \leq \underset{n \rightarrow \infty}{\limsup }\left\|y_{n}-p\right\| \leq d .
$$

Hence

$$
\lim _{n \rightarrow \infty}\left\|y_{n}-p\right\|-d
$$

Implies that 


$$
\lim _{n \rightarrow \infty}\left\|\beta_{n}\left(S^{n} x_{n}-p\right)+\left(1-\beta_{n}\right)\left(x_{n}-p\right)=d\right\|
$$

Using Lemma 2.2, we get

$$
\lim _{n \rightarrow \infty}\left\|x_{n}-S^{n} x_{n}\right\|=0 \text {. }
$$

Again

$$
\begin{aligned}
\left\|T^{n} x_{n}-x_{n}\right\| & \leq\left\|T^{n} x_{n}-T^{n} y_{n}\right\|+\left\|T^{n} y_{n}-x_{n}\right\| \\
& \leq k_{n}\left\|x_{n}-y_{n}\right\|+\left\|T^{n} y_{n}-x_{n}\right\| \\
& \leq k_{n}\left\|x_{n}-\left\{\left(1-\beta_{n}\right) x_{n}+\beta_{n} S^{n} x_{n}\right\}\right\|+\left\|T^{n} y_{n}-x_{n}\right\| \\
& \leq k_{n}\left\|x_{n}-S^{n} x_{n}\right\|+\left\|T^{n} y_{n}-x_{n}\right\|
\end{aligned}
$$

Implies together with (3.3) and (3.4) that

$$
\lim _{n \rightarrow \infty}\left\|x_{n}-T^{n} x_{n}\right\|=0 \lim _{n \rightarrow \infty}\left\|x_{n}-S^{n} x_{n}\right\|
$$

Applying Lemma 2.4 shows that

$$
\lim _{n \rightarrow \infty}\left\|x_{n}-T x_{n}\right\|=0 \lim _{n \rightarrow \infty}\left\|x_{n}-S x_{n}\right\|
$$

Theorem 3.2. Let $X$ a uniformly convex Banach space satisfying Opial's condition and let $\mathrm{D}, \mathrm{T}, \mathrm{S}$ and $\left\{x_{n}\right\}$ be as taken in Theorem 3.1. If $F(T) \cap F(S) \neq \theta$ then $\left\{x_{n}\right\}$ converges weakly to a common fixed point of $\mathrm{T}$ and. $\mathrm{S}$.

Proof. We prove that $\left\{x_{n}\right\}$ has a unique weak subsequential limit in $F(T) \cap F(S)$. To prove this, let $v$ and $v$ be weak limits of the subsequences $\left\{x_{n j}\right\}$ and $\left\{x_{n j}\right\}$ of $\left\{x_{n}\right\}$ respectively. By Theorem 3.1, $\lim _{n \rightarrow \infty}\left\|x_{n}-T x_{n}\right\|=0 \lim _{n \rightarrow \infty}\left\|x_{n}-S x_{n}\right\|$ and I-T,I-S are demiclosed with respect to zero by Lemma 2.3, we obtain that $T u=u$ and $S u=u$. Similarly, we can prove that $u \in F(T) \cap F(S)$. If $u \neq v$, then by Opial's condition.

$$
\begin{aligned}
\lim _{n \rightarrow \infty}\left\|x_{n}-u\right\| & =\lim _{n \rightarrow \infty}\left\|x_{n}-u\right\|<\lim _{n \rightarrow \infty}\left\|x_{n i}-u\right\| \\
& =\lim _{n \rightarrow \infty}\left\|x_{n}-v\right\|=\lim _{n \rightarrow \infty}\left\|x_{n j}-u\right\| \\
& <\lim _{n \rightarrow \infty}\left\|x_{n j}-u\right\|=\lim _{n \rightarrow \infty}\left\|x_{n}-u\right\|
\end{aligned}
$$

This is contradiction and hence the proof is complete.

Theorem 3.3. Let $X$ be uniformly convex Banach space and let $\mathrm{D}$ be a nonempty closed convex bounded subset of $\mathrm{X}$ which satisfying Opial's condition. Let $T, S: D \rightarrow D$ be a continuous mappings satisfying condition (1.2). Given a sequence as in (1.3), then $\left\{x_{n}\right\}$ converges weakly to a common fixed point of $\mathrm{T}$ and $\mathrm{S}$.

Proof. Since $p \in F(T) \cap F(S)$. Then

$$
\begin{aligned}
\left\|x_{n+1}-p\right\| & =\left\|\left(1-a_{n}\right) x_{n}+a_{n} T^{n} y_{n}-p\right\| \\
& \leq\left\|x_{n}-p\right\|+\left\|T^{n} y_{n}-p\right\|
\end{aligned}
$$

Using Lemma 2.6, we obtain

$$
\begin{aligned}
& \left\|x_{n+1}-p\right\| \leq\left\|x_{n}-p\right\|+\frac{a_{n}+c_{n}}{1-c_{n}}\left\|p-y_{n}\right\| \\
& \quad \leq\left\|x_{n}-p\right\|+\frac{a_{n}+c_{n}}{1-c_{n}}\left(\left\|p-x_{n}\right\|+\left\|p-S^{n} x_{n}\right\|\right)
\end{aligned}
$$


New, again using Lemma (2.6)

$$
\left\|x_{n+1}-p\right\| \leq\left\|x_{n}-p\right\|+\frac{a_{n}+c_{n}}{1-c_{n}}\left\|x_{n}-p\right\|+\left(\frac{a_{n}+c_{n}}{1-c_{n}}\right)^{2}\left\|x_{n}-p\right\|
$$

Let $\frac{a_{n}+c_{n}}{1-c_{n}}=L_{n}$. Then

$$
\left\|x_{n+1}-p\right\| \leq\left(1+L_{n}+L_{n}^{2}\right)\left\|x_{n}-p\right\|
$$

From Lemma 2.1, we get $\lim _{n \rightarrow \infty}\left\|x_{n}-p\right\|$ exists.

Let $\lim _{n \rightarrow \infty}\left\|x_{n}-p\right\|=d$ for some $\mathrm{d}>0$.

Since

$$
\begin{aligned}
\left\|y_{n}-p\right\| & =\left\|\left(1-\beta_{n}\right) x_{n}+\beta_{n} S^{n} x_{n}-p\right\| \\
& \leq\left\|x_{n}-p\right\|+L_{n}\left\|x_{n}-p\right\| .
\end{aligned}
$$

Now

$$
\underset{n \rightarrow \infty}{\limsup }\left\|y_{n}-p\right\| \leq \underset{n \rightarrow \infty}{\limsup }\left\|x_{n}-p\right\| \leq d \text {. }
$$

and

Then

$$
\left\|T^{n} y_{n}-p\right\|=L_{n}\left\|y_{n}-p\right\|
$$

$$
\underset{n \rightarrow \infty}{\limsup }\left\|T^{n} y_{n}-p\right\| \leq \underset{n \rightarrow \infty}{\limsup }\left\|x_{n}-p\right\| \leq d .
$$

Now

$$
\limsup _{n \rightarrow \infty}\left\|y_{n}-p\right\| \leq \limsup _{n \rightarrow \infty}\left\|x_{n}-p\right\| \leq d .
$$

Now consider, we have

$$
\lim _{n \rightarrow \infty}\left\|x_{n+1}-p\right\|=\lim _{n \rightarrow \infty}\left\|a_{n}\left(T^{n} y_{n}-p\right)+\left(1-a_{n}\right)\left(x_{n}-p\right)\right\| .
$$

From Lemma 2.2, we obtain

$$
\lim _{n \rightarrow \infty}\left\|x_{n}-T^{n} x_{n}\right\|=0 \text {. }
$$

Next

Note that

$$
\begin{aligned}
\left\|x_{n}-p\right\| & \leq\left\|x_{n}-T^{n} y_{n}\right\|+\left\|T^{n} y_{n}-p\right\| \\
& \leq\left\|x_{n}-T^{n} y_{n}\right\|+L_{n}\left\|y_{n}-p\right\| .
\end{aligned}
$$

$$
\|x n-p\| \leq \liminf _{n \rightarrow \infty}\|y n-p\| \leq \underset{n \rightarrow \infty}{\limsup }\left\|y_{n}-p\right\| \leq d .
$$

Hence

$$
\lim _{n \rightarrow \infty}\left\|y_{n}-p\right\|=d
$$

That is

$$
\lim \left\|\beta_{n}\left(S^{n} x_{n}-p\right)+\left(1-\beta_{n}\right)\left(x_{n}-p\right)\right\|=d .
$$

Since

$$
\underset{n \rightarrow \infty}{\limsup }\left\|S^{n} x_{n}-p\right\| \leq d \text { and } \underset{n \rightarrow \infty}{\limsup }\left\|x_{n}-p\right\| \leq d .
$$


From Lemma 2.2, we obtain

$$
\lim _{n \rightarrow \infty}\left\|x_{n}-S^{n} x_{n}\right\|=0
$$

Now, again

$$
\underset{n \rightarrow \infty}{\limsup }\left\|S^{n} x_{n}-p\right\| \leq d \text { and } \limsup _{n \rightarrow \infty}\left\|x_{n}-p\right\| \leq d .
$$

From Lemma 2.2, we get

$$
\lim _{n \rightarrow \infty}\left\|x_{n}-S^{n} x_{n}\right\|=0
$$

We obtain that

$$
\begin{aligned}
\left\|x_{n}-T^{n} x_{n}\right\| & \leq\left\|x_{n}-S^{n} y_{n}\right\|+\left\|S^{n} y_{n}-T^{n} x_{n}\right\| \\
& \leq\left\|x_{n}-S^{n} y_{n}\right\|+L_{n}\left\|x_{n}-y_{n}\right\|+\frac{2 c_{n}}{1-c_{n}}\left\|T^{n} x_{n}\right\| \\
& \leq \frac{1-c_{n}}{1-3 c_{n}}\left\{L_{n}\left\|x_{n}-S^{n} x_{n}\right\|+\left\|x_{n}-S^{n} y_{n}\right\|\right\}
\end{aligned}
$$

Implies together with (3.5) and (3.6) that

$$
\lim _{n \rightarrow \infty}\left\|x_{n}-S^{n} x_{n}\right\|=0=\lim _{n \rightarrow \infty}\left\|x_{n}-T^{n} x_{n}\right\| .
$$

Lemma 2.5 reveals that

$$
\lim _{n \rightarrow \infty}\left\|x_{n}-T x_{n}\right\|=0=\lim _{n \rightarrow \infty}\left\|x_{n}-S x_{n}\right\|
$$

The rest of the proof follows the lines similar to Theorem 3.2 and is therefore omitted. This completes the proof of the theorem.

Theorem 3.4. Let $\mathrm{D}$ be a nonempty compact convex subset of a uniformly convex Banach space $\mathrm{X}$ and T,S and $\left\{x_{n}\right\}$ as in Theorem 3.1. If $F(T) \cap F(S) \neq \theta$, then $\left\{x_{n}\right\}$ converges strongly to a common fixed point of $\mathrm{T}$ and $\mathrm{S}$.

Theorem 3.5. Let $\mathrm{D}$ be a nonempty compact convex subset of a uniformly convex Banach space $\mathrm{X}$ and let $T, S: D \rightarrow D$ be a continuous mappings satisfying condition (1.1). Given a sequence $\left\{x_{n}\right\}$ as in (1.3), the $\left\{x_{n}\right\}$ converges strongly to a common fixed point of $\mathrm{T}$ and $\mathrm{S}$.

Remark 3.6 Theorem 3.4 and Theorem 3.5 generlaize the results of Khan and Takahashi [3, Theorem 2].

\section{CONCLUSIONS}

The study of the approximation of common fixed points for more general classes of mappings through weak and strong convergence results of an iterative scheme in a uniformly convex Banach space shows that $T, S: D \rightarrow D$ be an asymptotically quasi-nonexpansive mappings with sequence $\left\{k_{n}\right\}$ such that $\sum_{n=1}^{\infty}\left(k_{n}-1\right)<\infty$ and $F(T) \cap F(S) \neq \phi$.

\section{REFERENCES}

[1] G.Das and J.P.Debata, Fixed points of quasi-nonexpansive mappings, Indian J.Pure Appl. Math., 17 (1986), 1263-1269.

[2] S.H.Khan and W.Takahashi, Iterative approximation of fixed point of asymptotically non-expansive mappings with compact, domain, to appear in "Pan American Mathematical Journal". 
[3] S.H.Khan and W. Takahashi, Approximating common fixed points of two asymptotically nonexpansive mappings, Sci. Math. Japon, 53 (1) (2001), 143-148.

[4] W.Takhashi and T.Tamura, Covergence theorems for a pair of nonexpansive mappings, J.Convex Anal., 5(1) (1995), 45-58.

[5] S.C.Bose and S.K. Laskar, Fixed theorems for certain class of mappings, J.Math. Phy. Sci., 19 (1983), 503-509.

[6] Q.Liu, Iterative sequences for asymptotically quasi-nonexpansive mappings, J.Math.Anal.Appl.,259 (1) (2001), 1-7.

[7] K.Goebel and W.A. Kirk, A fixed points theorem for asymptotically nonexpansive mappings, Proc.Amer. Math.Soc., 35(1) (1972), 171-174.

[8] K.K.Tan and H.K.Xu, Fixed points iteration process for asymptotically nonexpansive mappings, Proc, Amer. Math. Soc., 122 (1994), 733-739.

[9] J.Schu, Weak and strong convergence to fixed points for asymptotically nonexpansive mappings, Bull. Austral. Math., Soc., 43 (1991), 153-159.

[10] J.Schu, Iterative construction of fixed points of asymptotically nonexpansive mappings, J.Math.Anal.Appl., 158 (1991), 407-413.

[11] J.Gornicki, Weak convergence theorems for asymptotically nonexpansive mappings in uniformly convex Banach spaces, Comment. Math. Univ. Garolin., 30 (1989), 249- 252.

[12] Z.Opial, Weak convergence of the sequence of successive approximations of nonexpansive mappings, Bull.Amer. Math. Soc., 73 (1967), 591-597.

[13] H.F. Senter and W.G.Dotson, Approximating fixed points of nonexpansive mappings, mer. Math. Soc., 44 (2) (1974), 375-380. 